\title{
REMARKS ON THE ROLE OF PULSARS \\ IN COSMIC RAY PRODUCTION
}

\author{
V. L. GINZBURG \\ P. N. Lebedev Institute of Physics, Soviet Academy of Sciences, Moscow, U.S.S.R.
}

This discussion is concerned with the production of cosmic rays in supernova explosions. In particular, we are discussing the role of pulsars (which we all believe to be neutron stars) in this process. I should like first to recall that (so far as I am aware) the idea that neutron stars form in supernova explosions, and that this phenomenon is relevant to cosmic rays, was first proposed by Baade and Zwicky in 1934. The argument was originally based on energy considerations, and further estimates indicated that supernovae could indeed be the main source of cosmic rays (ter Haar, 1950). When radioastronomical data later showed that supernova remnants definitely contained large numbers of relativistic electrons, there could be no doubt that they were sources of cosmic rays as well (and it became quite probable that they were indeed the major source of such particles). The growth of these ideas can be studied in historical perspective in Rosen's (1969) compilation of papers on cosmic ray origin theories. As is so often the case, this subject has developed in a cyclic and repetitious fashion. I wish to make this point because I feel that my own contribution to these theories has sometimes been overestimated - though I hope I cannot myself be blamed for this.

It would not be appropriate to consider cosmic ray origins in detail during this discussion. I have in my case given my present views elsewhere (Ginzburg, 1969, 1970). The following remarks will deal specifically with the role of pulsars.

To prove that supernovae are the main sources of the cosmic rays observed near the Earth, one must show that:

(a) The energy balance is fulfilled - this requires that supernovae inject cosmic rays into the Galaxy with an average power $U=10^{40}-10^{41} \mathrm{erg} / \mathrm{sec}^{-1}$.

(b) Supernovae accelerate protons and heavy nuclei in a manner such that their total energy is two orders of magnitude greater than that in electrons. The nuclei must have an energy and charge spectrum in accord with the observations, and the electrons must also have the appropriate energy spectrum.

(c) Most of our evidence on cosmic ray acceleration in supernovae is based on the Crab Nebula. We must therefore understand the relation of the Crab to other supernova remnants. (Possibly it is an exceptional source).

(d) The role of other possible cosmic ray sources must be clarified. I have in mind the galactic nucleus, novae, magnetic white dwarfs, etc.

(All these questions have been under consideration for several years. See Ginzburg $(1969,1970)$ and the literature cited therein.)

Let us now ask whether the discovery of pulsars has changed the situation in respect of any of these problems. The answer seems to me absolutely negative. This 
is plain if one recalls our limited present knowledge of cosmic ray acceleration in or near the pulsars. We know neither the total energy, nor the energy and charge spectra, of the accelerated particles. The remarks by Dr Cameron at this discussion further aggravate the difficulty of obtaining the correct charge spectra. I should like also to stress the necessity to take account of energy losses, and (in the case of nuclei) transmutation of particles, in the vicinity of pulsars. A further problem - and perhaps the most crucial for pulsar theories in general - is to allow for plasma effects near the neutron star in a self consistent manner.

I am therefore convinced that, at the present time, the discovery of pulsars does not change our general picture of the problems of cosmic ray origin (and particularly the supernova origin theory). But this statement is, most emphatically, not intended in any way to minimize the importance of the discovery of these objects - my Invited Discourse (this volume, page 20) makes it abundantly clear that I hold quite the contrary view. Moreover, this statement does not contradict the belief that pulsars are in fact quite relevant to cosmic ray origins.

Previously, the generation of cosmic rays in supernovae was associated with the stellar explosion, or with the supernova shell. It is true that the possibility of active supernova remnants had been raised before pulsars were known, but at that stage no quantitative estimates were possible. The discovery of pulsars has really demonstrated the existence of a third 'channel' for cosmic ray generation in supernovae. I agree also with Dr Ramaty that pulsars, as indicators of old supernova remnants, provide extra information about the distribution of cosmic ray sources. Finally, acceleration near pulsars can, in principle, yield particles with very high energies, and this is important in connexion with the origin of particles with $E \gtrsim 10^{17}-10^{18} \mathrm{eV}$ (for more details, see Ginzburg, 1969). In both these cases, however, one is speaking of future possibilities rather than present achievements.

So, there is really no foundation for the view, often expressed in the literature, that the discovery of pulsars has already drastically changed our perspectives on the origin of cosmic rays. I think such an opinion is based on a misunderstanding, and cannot be shared by anyone who is really informed about the present day status of our knowledge of these matters.

\section{References}

Baade, W. and Zwicky, F.: 1934a, Proc. Nat. Acad. Sci. 20, 259.

Baade, W. and Zwicky, F.: 1934b, Phys. Rev. 46, 76.

Ginzburg, V. L.: 1969, Comm. Astrophys. Space Phys. 1, 207.

Ginzburg, V. L.: 1970, Comm. Astrophys. Space Phys. 2, 1, 43.

Rosen, S.: 1969 (ed.), Selected Papers on Cosmic Ray Origin Theories, Dover Publications, New York.

ter Haar, D.: 1950, Rev. Mod. Phys. 22, 119. 


\section{COMMENTS}

T. Gold: I quite agree with Dr Ginzburg that there are many things that we don't know, but on the other hand I think he was a little negative on what the pulsars have in fact given us. It is not that we merely see the supernova shell to have fast particles in it. We know in the Crab Nebula that the fast particles that we see in the shell have to be currently produced because they lose their energy into light in a time very short compared with the time since the explosion of the object. So therefore it is clear that there is an acceleration mechanism at work in the Nebula at the present time. That is the mechanism that has to supply most of the radiated energy of the Crab Nebula. That is the famous $10^{38} \mathrm{erg}$. So we do know that something has been producing energetic particles. Most of the energy that we now see is currently produced through energetic particles in the Crab Nebula and we therefore are very glad to see an object that could be the source. Until the pulsar was discovered one did not know what that source could be.

Secondly, in the radiation mechanism, as I tried to stress, we do observe that high gamma particles indeed are present in the close vicinity of the pulsar. So we have seen, without question, a cosmic synchrotron at work. It is making at least $10^{13} \mathrm{eV}$ particles and that is a very good beginning, it seems to me, for trying to study the origin of cosmic rays. At least it is a better beginning than if we merely had to confine ourselves to a theoretical discussion of objects that we have never been able to investigate in detail at all.

The other point is that the rotational energy of a pulsar is of the same order, only a few times less, than the entire explosion. So therefore, the question is which is more efficient in making fast particles. Now in the explosion one discusses that one may get a few percent of the energy, through a steepening of a shockwave, as discussed by Colgate, into high energy particles. The rotational energy of a spinning magnet may well be, for all that I know, a hundred percent efficient or very close to that, for conversion into fast particles. Since we are only a few times down in energy in the rotation, as compared with the explosion, the rotation may win since a larger fraction may go into high energy particles.

The last point I want to make is the question of whether the $10^{52} \mathrm{erg}$ in the early phases will blow away the supernova shell. If you deposited $10^{52} \mathrm{erg}$ in the surrounding shell, then of course it would be blown away with much higher velocity that it now is seen to possess. But on the other hand if the situation is that these high energy particles are put chiefly into a plane or into some set of particular directions, as is very likely the case, then the whole shell would not be blown away. It may well be that the flow in any case is Taylor unstable, so that the whole massive shell is not able to catch the outward going momentum that is going into the very high energy particles. I cannot be persuaded that it is impossible that the high energy particles could have come out of the system and yet that over some areas a shell would be left which later, in turbulent motion, will knit itself together in any way you like. So I don't really believe we can exclude the possibility that the rotational energy comes out as particles in the early phases; but I am willing to admit that we can't prove that point yet. 